https://doi.org/10.48048/tis.2021.1443

\title{
Neutrosophic Orbit Topological Spaces
}

\section{T Madhumathi* and F NirmalaIrudayam}

Department of Mathematics, Nirmala College for Women, Coimbatore, India

('Corresponding author's e-mail: madhumanoj1822@gmail.com)

Received: 8 February 2021, Revised: 23 June 2021, Accepted: 8 July 2021

\begin{abstract}
Neutrosophy is a flourishing arena which conceptualizes the notion of true, falsity and indeterminancy attributes of an event. In the study of dynamical systems, an orbit is a collection of points related by the evolution function of the dynamical system. Hence in this paper we focus on introducing the concept of neutrosophic orbit topological space denoted as $\left(X, \tau_{N O}\right)$. Also, some of the important characteristics of neutrosophic orbit open sets are discussed with suitable examples.
\end{abstract}

Keywords: Neutrosophic orbit open set, Neutrosophic orbit topology, Neutrosophic orbit topological spaces

\section{Introduction}

Fuzzy concept has invaded almost all branches of Mathematics since its introduction of the concept of fuzzy set by Zadeh [14]. Fuzzy sets have applications in many fields. The idea of fuzzy set is welcomed because it handles uncertainty and vagueness which Cantorian set could not address. However, in reality, it may not always be true that the degree of non-membership of an element in a fuzzy set is equal to 1 minus the membership degree because there may be some hesitation degree. Therefore, a generalization of fuzzy sets was introduced byAtanassov [1] as intuitionistic fuzzy sets (IFS) which incorporated the degree of hesitation called hesitation margin (and is defined as 1 minus the sum of membership and non-membership degrees respectively). As a generalization of intuitionistic fuzzy sets neutrosophic set was formulated by Smarandache [8-10] originally gave the definition of a neutrosophic set and neutrosophic logic. The neutrosophic logic is a formal frame trying to measure the truth, indeterminacy and falsehood. In 2012 Salama and Alblowi [11,12] introduced the concept of neutrosophic topological spaces(NTOP). The concept of orbit function in general metric space was introduced by Devaney [2]. The orbit in mathematics has an important role in the study of dynamical systems. The concept of fuzzy orbit open sets under the mapping $f: X \rightarrow X$ in a fuzzy topological space $(X, \tau)$ was introduced by Malathi and Uma[4]. The concept of fuzzy orbit topological spaces was introduced by Majeed and El-Sheikh[5]. The concept of intuitionistic fuzzy orbit set and intuitionistic fuzzy orbit topological space was introduced by Priscilla and Irudayam [3]. The concept of neutrosophic orbit set was introduced by Madhumathi et al. [6]. The purpose of this paper is to study the collection of all neutrosophic orbit open sets under the mapping $f: X \rightarrow X$. we introduce the necessary conditions on the mapping $\boldsymbol{f}$ in order to obtain a fixed orbit of a neutrosophic set (i.e., $\boldsymbol{f}(\boldsymbol{\mu})=\boldsymbol{\mu})$ for any neutrosophic orbit open set $\boldsymbol{\mu}$ under the mapping $\boldsymbol{f}$. Also, some properties of neutrosophic orbit open sets related with union (intersection) of these sets are introduced. we also prove that the family of all neutrosophic orbit open sets constructs a new neutrosophic topological space. This new space is called neutrosophic orbit topological space $\left(X, \tau_{N O}\right)$. Furthermore, the concept of neutrosophic orbit interior (closure) is defined. Finally, the category of neutrosophic orbit topological spaces (NOTOP) is defined. And we show this category is isomorphic to a subcategory of the category of NTOP. 


\section{Preliminaries}

1 Definition [9] Let $X$ be a nonempty set. A neutrosophic set (NS for short) $A$ is an object having the form $\mathrm{A}=<\mathrm{x}, \mathrm{A}^{\mathrm{T}}, \mathrm{A}^{\mathrm{I}}, \mathrm{A}^{\mathrm{F}}>$ where $\mathrm{A}^{\mathrm{T}}, \mathrm{A}^{\mathrm{I}}, \mathrm{A}^{\mathrm{F}}$ represent the degree of membership, the degree of indeterminacy and the degree of non-membership respectively of each element $\mathrm{x} \in \mathrm{X}$ to the set $\mathrm{A}$.

2 Definition [9] Let $\mathrm{X}$ be a non empty set, $\mathrm{A}=<\mathrm{x}, \mathrm{A}^{\mathrm{T}}, \mathrm{A}^{\mathrm{I}}, \mathrm{A}^{\mathrm{F}}>$ and $\mathrm{B}=<\mathrm{x}, \mathrm{B}^{\mathrm{T}}, \mathrm{B}^{\mathrm{I}}, \mathrm{B}^{\mathrm{F}}>$ be neutrosophic sets on $\mathrm{X}$, and let $\left\{\mathrm{A}_{\mathrm{i}}: \mathrm{i} \in \mathrm{J}\right\}$ be an arbitrary family of neutrosophic sets in $\mathrm{X}$, where $\mathrm{A}_{\mathrm{i}}=<\mathrm{X}, \mathrm{A}^{\mathrm{T}}, \mathrm{A}^{\mathrm{I}}, \mathrm{A}^{\mathrm{F}}>$

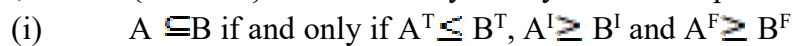

(ii) $\quad \mathrm{A}=\mathrm{B}$ if and only if $\mathrm{A} \leq \mathrm{B}$ and $\mathrm{B} \leq \mathrm{A}$.

(iii) $\bar{A}=<\mathrm{x}, \mathrm{A}^{\mathrm{F}}, 1-\mathrm{A}^{\mathrm{I}}, \mathrm{A}^{\mathrm{T}}>$

(iv) $\mathrm{A} \cap \mathrm{B}=<\mathrm{x}, \mathrm{A}^{\mathrm{T}} \wedge \mathrm{B}^{\mathrm{T}}, \mathrm{A}^{\mathrm{I}} \vee \mathrm{B}^{\mathrm{I}}, \mathrm{A}^{\mathrm{F}} \vee \mathrm{B}^{\mathrm{F}}>$

(v) $\quad \mathrm{A} \cup \mathrm{B}=<\mathrm{x}, \mathrm{A}^{\mathrm{T}} \vee \mathrm{B}^{\mathrm{T}}, \mathrm{A}^{\mathrm{I}} \wedge \mathrm{B}^{\mathrm{I}}, \mathrm{A}^{\mathrm{F}} \wedge \mathrm{B}^{\mathrm{F}}>$

(vi) $\quad \mathrm{UA}_{\mathrm{i}}=<\mathrm{x}, \mathrm{VA}_{\mathrm{i}}^{\mathrm{T}}, \wedge \mathrm{A}_{\mathrm{i}}{ }^{\mathrm{I}}, \wedge \mathrm{A}_{\mathrm{i}}{ }^{\mathrm{F}}>$

(vii) $\quad \cap \mathrm{A}_{\mathrm{i}}=<\mathrm{x}, \wedge \mathrm{A}_{\mathrm{i}}^{\mathrm{T}}, \mathrm{VA}_{\mathrm{i}}^{\mathrm{I}}, \mathrm{VA}_{\mathrm{i}}^{\mathrm{F}}>$

(viii) $\mathrm{A}-\mathrm{B}=\mathrm{A} \wedge \bar{B}$.

(ix) $\quad 0_{\mathrm{N}}=<\mathrm{x}, 0,1,1>; 1_{\mathrm{N}}=<\mathrm{x}, 1,0,0>$.

3 Definition [11] A neutrosophic topology (NT for short) on a nonempty set $\mathrm{X}$ is a family $\tau$ of neutrosophic set in $\mathrm{X}$ satisfying the following axioms:

(i) $0_{\mathrm{N}}, 1_{\mathrm{N}} \in \tau$.

(ii) $\mathrm{G}_{1} \wedge \mathrm{A}_{2} \in \tau$ for any $\mathrm{G}_{1}, \mathrm{G}_{2} \in \tau$.

(iii) $\vee \mathrm{G}_{\mathrm{i}} \in \tau$ for any arbitrary family $\left\{\mathrm{G}_{\mathrm{i}}: \mathrm{i} \in \mathrm{J}\right\} \subseteq \tau$.

In this case the pair $(\mathrm{X}, \tau)$ is called a neutrosophic topological space (NTS for short) and any neutrosophic set in $\tau$ is called a neutrosophic open set(NOS for short) in X. The complement A of a neutrosophic open set A is called a neutrosophic closed set (NCS for short) in X.

4 Definition [11] Let $(X, \tau)$ be a neutrosophic topological space and $A=<X, A^{T}, A^{I}, A^{F}>$ be a set in $X$. Then the closure and interior of $\mathrm{A}$ are defined by

$\operatorname{Ncl}(\mathrm{A})=\Lambda\{\mathrm{K}: \mathrm{K}$ is a neutrosophic closed set in $\mathrm{X}$ and $\mathrm{A} \leq \mathrm{K}\}$,

$\operatorname{Nint}(A)=V\{G: G$ is a neutrosophic open set in $X$ and $G \leq A\}$.

It can be also shown that $\mathrm{Ncl}(\mathrm{A})$ is a neutrosophic closed set and $\operatorname{Nint}(\mathrm{A})$ is a neutrosophic open set in $\mathrm{X}$, and $\mathrm{A}$ is a neutrosophic closed set in $\mathrm{X}$ iff $\operatorname{Ncl}(\mathrm{A})=\mathrm{A}$; and $\mathrm{A}$ is a neutrosophic open set in $\mathrm{X}$ iff $\operatorname{Nint}(\mathrm{A})$ $=\mathrm{A}$.

5 Definition [2] Orbit of a point $x$ in $X$ under the mapping $f$ is $O_{f}(x)=\left\{x, f(x), f^{2}(x), \ldots\right\}$

6 Definition [6] Let $\mathrm{X}$ be a nonempty set and $\mathrm{f}: \mathrm{X} \rightarrow \mathrm{X}$ be any mapping. Let $\alpha$ be any neutrosophic set in $\mathrm{X}$. The neutrosophic orbit $\mathrm{O}_{\mathrm{f}}(\alpha)$ of $\alpha$ under the mapping $\mathrm{f}$ is defined as $\mathrm{O}_{\mathrm{fT}}(\alpha)=\left\{\alpha, \mathrm{f}^{\mathrm{l}}(\alpha), \mathrm{f}^{2}(\alpha), \ldots \mathrm{f}^{\mathrm{n}}(\alpha)\right\}$, $\mathrm{O}_{\mathrm{fl}}(\alpha)=\left\{\alpha, \mathrm{f}^{\mathrm{l}}(\alpha), \mathrm{f}^{2}(\alpha), \ldots \mathrm{f}^{\mathrm{n}}(\alpha)\right\}, \mathrm{O}_{\mathrm{fF}}(\alpha)=\left\{\alpha, \mathrm{f}^{\mathrm{l}}(\alpha), \mathrm{f}^{2}(\alpha), \ldots \mathrm{f}^{\mathrm{n}}(\alpha)\right\}$ for $\alpha \in \mathrm{X}$ and $\mathrm{n} \in \mathrm{Z}^{+}$.

7 Definition [6] Let $\mathrm{X}$ be a nonempty set and let $\mathrm{f}: \mathrm{X} \rightarrow \mathrm{X}$ be any mapping. The neutrosophic orbit set of $\alpha$ under the mapping $\mathrm{f}$ is defined as $\mathrm{NO}_{\mathrm{f}}(\alpha)=<\alpha, \mathrm{O}_{\mathrm{fT}}(\alpha), \mathrm{O}_{\mathrm{fl}}(\alpha), \mathrm{O}_{\mathrm{fF}}(\alpha)>$ for $\alpha \in \mathrm{X}$, where $\mathrm{O}_{\mathrm{fT}}(\alpha)=$ $\left\{\alpha \wedge \mathrm{f}^{1}(\alpha) \wedge \mathrm{f}^{2}(\alpha) \wedge \ldots \wedge \mathrm{f}^{\mathrm{n}}(\alpha)\right\}, \mathrm{O}_{\mathrm{fl}}(\alpha)=\left\{\alpha \vee f^{1}(\alpha) \vee f^{2}(\alpha) \vee \ldots \vee f^{\mathrm{n}}(\alpha)\right\}, \mathrm{O}_{\mathrm{fF}}(\alpha)=\left\{\alpha \vee f^{1}(\alpha) \vee f^{2}(\alpha) \vee \ldots \vee f^{\mathrm{n}}(\alpha)\right\}$.

8 Definition [6] Let $(\mathrm{X}, \tau)$ be a neutrosophic topological space. Let $\mathrm{f}: \mathrm{X} \rightarrow \mathrm{X}$ be any mapping. The neutrosophic orbit set under the mapping $\mathrm{f}$ which is in neutrosophic topology $\tau$ is called neutrosophic orbit open set under the mapping f. Its complement is called a neutrosophic orbit closed set under the mapping $f$.

9 Example Let $\mathrm{X}=\{\mathrm{a}, \mathrm{b}, \mathrm{c}\}=\mathrm{Y}$. Define $\tau=\left\{0_{\mathrm{N}}, 1_{\mathrm{N}}, \alpha, \gamma\right\}$ where $\left.\alpha^{T}, \gamma^{T}: X \rightarrow\right]^{-} 0,1^{+}[$

$\left.\alpha^{I}, \gamma^{I}: X \rightarrow\right]^{-} 0,1^{+}[$

$\left.\alpha^{F}, \gamma^{F}: X \rightarrow\right]^{-} 0,1^{+}[$

are defined as

$\alpha^{T}(a)=0.3, \alpha^{I}(a)=0.5, \alpha^{F}(a)=0.6, \alpha^{T}(b)=0.4, \alpha^{I}(b)=0.6, \alpha(b)=0.8, \alpha^{T}(c)=0.1$,

$\alpha^{I}(c)=0.3, \alpha^{F}(c)=0.7$

$\gamma^{T}(a)=0.1, \gamma^{I}(a)=0.6, \gamma^{F}(a)=0.8, \gamma^{T}(b)=0.1, \gamma^{I}(b)=0.6, \gamma^{F}(b)=0.8$,

$\gamma^{T}=0.1, \gamma^{I}(c)=0.6, \gamma^{F}(c)=0.8$. 
Define $\mathrm{f}: \mathrm{X} \rightarrow \mathrm{X}$ as $\mathrm{f}(\mathrm{a})=\mathrm{c}, \mathrm{f}(\mathrm{b})=\mathrm{a}, \mathrm{f}(\mathrm{c})=\mathrm{b}$. The neutrosophic orbit set of $\alpha$ under the mapping $\mathrm{f}$ is defined as $\mathrm{NO}_{\mathrm{f}}(\alpha)=\alpha \cap \mathrm{f}^{1}(\alpha) \cap \mathrm{f}^{2}(\alpha) \cap \ldots \cap \mathrm{f}^{\mathrm{n}}(\alpha), \mathrm{NO}_{\mathrm{f}}(\alpha)=\gamma$. Then $\gamma$ is a neutrosophic orbit open set under the mapping $f$.

From the above definition its clear that every neutrosophic orbit open set under the mapping $\mathrm{f}$ is an open neutrosophic set in $\mathrm{X}$. But the converse is not true, in this example the neutrosohic set $\alpha$ is an open neutrosophic set, however it is not neutrosophic orbit open set under the mapping $\mathrm{f}$, because there is not exists a neutrosophic set $\vartheta \in I^{X}$ such that $\mathrm{NO}_{\mathrm{f}}(\vartheta)=\alpha$.

10 Definition [13] A mapping $f:(X, \tau) \rightarrow(Y, \tau)$ is called neutrosophic continuous if the inverse image of every closed set in $\mathrm{Y}$ is neutrosophic closed in $\mathrm{X}$.

\section{Some properties of neutrosophic orbit open sets}

In our work we consider $\mathrm{X}$ as a nonempty countable set,we give the conditions on a mapping $\mathrm{f}: \mathrm{X} \rightarrow$ $X$,to obtain a fixed neutrosophic orbit open $\operatorname{set}(\mathrm{i} . \mathrm{e} . \mathrm{f}(\mu)=\mu$ ) for any neutrosophic orbit open set $\mu$, and study some properties of these sets.

Theorem 3.1:

Let $(X, \tau)$ be a NTOP and $f: X \rightarrow X$ be any bijective mapping. Then $f(\mu)=\mu$ for any neutrosophic orbit open set $\mu$ under the mappingf.

Proof:

Let $(X, \tau)$ be a NTOPand $\mathrm{f}: \mathrm{X} \rightarrow \mathrm{X}$ be a bijective mapping. Then we have 3 cases:

Case 1:

If $f\left(x_{i}\right)=x_{j} ; x_{i}, x_{j} \in$ Xandi $\neq$ jforalli, $\mathrm{j} \in \wedge$.Suppose $X=\left\{x_{1}, x_{2}\right\}$ andf: $X \rightarrow$ Xdefined as

$f\left(x_{1}\right)=x_{2}, f\left(x_{2}\right)=x_{1}$. Let $\mu$ be a neutrosophic orbit open set under the mapping $f$.

Then there exists a neutrosophic set $\lambda \in \mathrm{I}^{\mathrm{X}}$ suchthatNO $_{\mathrm{f}}(\lambda)=\lambda \cap \mathrm{f}(\lambda) \cap \mathrm{f}^{2}(\lambda) \ldots=\mu$.

Let $\lambda=\left\{\left(\mathrm{x}_{1}, \mathrm{u}_{1}, \mathrm{v}_{1}, \mathrm{w}_{1}\right),\left(\mathrm{x}_{2}, \mathrm{u}_{2}, \mathrm{v}_{2}, \mathrm{w}_{2}\right) ; \mathrm{x}_{1}, \mathrm{x}_{2} \in \mathrm{X}, \mathrm{u}_{1}, \mathrm{u}_{2}, \mathrm{v}_{1}, \mathrm{v}_{2}, \mathrm{w}_{1}, \mathrm{w}_{2} \in \mathrm{I}\right\}$. This implies

$\mathrm{f}(\lambda)=\left\{\left(\mathrm{x}_{1}, \mathrm{u}_{2}, \mathrm{v}_{2}, \mathrm{w}_{2}\right),\left(\mathrm{x}_{2}, \mathrm{u}_{1}, \mathrm{v}_{1}, \mathrm{w}_{1}\right)\right\}, \mathrm{f}^{2}(\lambda)=\left\{\left(\mathrm{x}_{1}, \mathrm{u}_{1}, \mathrm{v}_{1}, \mathrm{w}_{1}\right),\left(\mathrm{x}_{2}, \mathrm{u}_{2}, \mathrm{v}_{2}, \mathrm{w}_{2}\right)\right\}, \ldots$

$\therefore \mathrm{NO}_{\mathrm{f}}(\lambda)=\left\{\left(\mathrm{x}_{1},\left[\inf \left(\mathrm{u}_{1}, \mathrm{u}_{2}, \mathrm{u}_{1}, \ldots\right), \sup \left(\mathrm{v}_{1}, \mathrm{v}_{2}, \mathrm{v}_{1}, \ldots\right), \sup \left(\mathrm{w}_{1}, \mathrm{w}_{2}, \mathrm{w}_{1}, \ldots\right)\right]\right.\right.$,

$\left(\mathrm{x}_{2},\left[\inf \left(\mathrm{u}_{2}, \mathrm{u}_{1}, \mathrm{u}_{2}, \ldots\right), \sup \left(\mathrm{v}_{2}, \mathrm{v}_{1}, \mathrm{v}_{2}, \ldots\right), \sup \left(\mathrm{w}_{2}, \mathrm{w}_{1}, \mathrm{w}_{2}, \ldots\right)\right]\right\}$

$=\left\{\left(\mathrm{x}_{1},\left[\min \left\{\mathrm{u}_{1}, \mathrm{u}_{2}\right\}, \max \left\{\mathrm{v}_{1}, \mathrm{v}_{2}\right\}, \max \left\{\mathrm{w}_{1}, \mathrm{w}_{2}\right\}\right]\right.\right.$,

$\left.\left(\mathrm{x}_{2},\left[\min \left\{\mathrm{u}_{1}, \mathrm{u}_{2}\right\}, \max \left\{\mathrm{v}_{1}, \mathrm{v}_{2}\right\}, \max \left\{\mathrm{w}_{1}, \mathrm{w}_{2}\right)\right\}\right]\right\}=\mu$

In general, if $X=\left\{\mathrm{x}_{1}, \mathrm{x}_{2}, \ldots\right\}$ and $\mu$ be a neutrosophic orbit open set under the mapping $\mathrm{f}$, then there exists a neutrosophic set

$\lambda=\left\{\left(\mathrm{x}_{1}, \mathrm{u}_{1}, \mathrm{v}_{1}, \mathrm{w}_{1}\right),\left(\mathrm{x}_{2}, \mathrm{u}_{2}, \mathrm{v}_{2}, \mathrm{w}_{2}\right),\left(\mathrm{x}_{3}, \mathrm{u}_{3}, \mathrm{v}_{3}, \mathrm{w}_{3}\right) \ldots \ldots\right\}=\left\{\left(\mathrm{x}_{\mathrm{i}}, \mathrm{u}_{\mathrm{i}}, \mathrm{v}_{\mathrm{i}}, \mathrm{w}_{\mathrm{i}}\right) ; \mathrm{x}_{\mathrm{i}} \in \mathrm{X}, \mathrm{u}_{\mathrm{i}}, \mathrm{v}_{\mathrm{i}}, \mathrm{w}_{\mathrm{i}} \in \mathrm{I}, \mathrm{i} \in \wedge\right\}$

Such that $\mathrm{NO}_{\mathrm{f}}(\lambda)=\mu$. That means

$\mathrm{NO}_{\mathrm{f}}(\lambda)=\left\{\left(\mathrm{x}_{\mathrm{i}},\left[\inf \left\{\mathrm{u}_{\mathrm{i}}\right\}, \sup \left\{\mathrm{v}_{\mathrm{i}}\right\}, \sup \left\{\mathrm{w}_{\mathrm{i}}\right\}\right]\right) ; \mathrm{x}_{\mathrm{i}} \in \mathrm{X}, \mathrm{u}_{\mathrm{i}}, \mathrm{v}_{\mathrm{i}}, \mathrm{w}_{\mathrm{i}} \in \mathrm{I}, \mathrm{i} \in \Lambda\right\}$

$=\left\{\left(\mathrm{x}_{\mathrm{i}},[\mathrm{r}, \mathrm{s}, \mathrm{t}]\right) ; \mathrm{x}_{\mathrm{i}} \in \mathrm{X},\left\{\mathrm{r}=\inf \left\{\mathrm{u}_{\mathrm{i}}\right\}, \mathrm{s}=\sup \left\{\mathrm{v}_{\mathrm{i}}\right\}, \mathrm{t}=\sup \left\{\mathrm{w}_{\mathrm{i}}\right\} ; \mathrm{u}_{\mathrm{i}}, \mathrm{v}_{\mathrm{i}}, \mathrm{w}_{\mathrm{i}} \in \mathrm{I}\right\}, \mathrm{i}=\mu\right.$

Now for eachx $x_{j} \in X$, we have

$\mathrm{f}(\mu)\left(x_{j}\right)= \begin{cases}\bigcup_{f\left(x_{i}\right)=x_{j}} \mu\left(x_{i}\right) & \text { if } f^{-1}\left(x_{j}\right) \neq \emptyset \\ (0,1,1) & \text { if } f^{-1}\left(x_{j}\right)=\varnothing\end{cases}$

From the hypothesis and the definition of $f$,we get $f(\mu)\left(x_{j}\right)=\mu\left(x_{i}\right)=r, s, t$ for all $x_{j} \in X$. Hence $f(\mu)=$ $\mu$.

Case 2:

If $f\left(x_{i}\right)=x_{j} ; x_{i}, x_{j} \in X$ and $i=j$ for some $i, j \in \wedge$. In this case the least number of elements in $X$ must be 3 elements. So,Suppose that if $X=\left\{x_{1}, x_{2}, x_{3}\right\}$, then from the hypothesis and the definition of $f$, the mapping $f: X \rightarrow X$ can be defined as $f\left(x_{1}\right)=x_{1}, f\left(x_{2}\right)=x_{3}$ and $f\left(x_{3}\right)=x_{2}\left(i . e ., f\left(x_{i}\right)=x_{j}\right.$ when $i=j=$ 1 and $f\left(x_{i}\right)=x_{j}, i \neq j$ when $\left.i, j \in\{2,3\}\right)$. Let $\mu$ be a neutrosophic orbit open set under the mapping $f$. Then there exists a neutrosophic set $\lambda \in \mathrm{I}^{\mathrm{X}}$ such that $\mathrm{NO}_{\mathrm{f}}(\lambda)=\lambda \cap \mathrm{f}(\lambda) \cap \mathrm{f}^{2}(\lambda) \ldots=\mu$.

Let $\lambda=\left\{\left(\mathrm{x}_{1}, \mathrm{u}_{1}, \mathrm{v}_{1}, \mathrm{w}_{1}\right),\left(\mathrm{x}_{2}, \mathrm{u}_{2}, \mathrm{v}_{2}, \mathrm{w}_{2}\right),\left(\mathrm{x}_{3}, \mathrm{u}_{3}, \mathrm{v}_{3}, \mathrm{w}_{3}\right) ; \mathrm{x}_{\mathrm{i}} \in \mathrm{X}, \mathrm{u}_{\mathrm{i}}, \mathrm{v}_{\mathrm{i}}, \mathrm{w}_{\mathrm{i}} \in \mathrm{I}, \mathrm{i}=1,2,3\right\}$.Then from the definition of $f$, we get

$f(\lambda)=\left\{\left(x_{1}, u_{1}, v_{1}, w_{1}\right),\left(x_{2}, u_{3}, v_{3}, w_{3}\right),\left(x_{3}, u_{2}, v_{2}, w_{2}\right)\right\}$,

$\mathrm{f}^{2}(\lambda)=\left\{\left(\mathrm{x}_{1}, \mathrm{u}_{1}, \mathrm{v}_{1}, \mathrm{w}_{1}\right),\left(\mathrm{x}_{2}, \mathrm{u}_{2}, \mathrm{v}_{2}, \mathrm{w}_{2}\right),\left(\mathrm{x}_{3}, \mathrm{u}_{3}, \mathrm{v}_{3}, \mathrm{w}_{3}\right)\right\} \ldots$

$\therefore \mathrm{NO}_{\mathrm{f}}(\lambda)=\left(\mathrm{x}_{1}, \mathrm{u}_{1}, \mathrm{v}_{1}, \mathrm{w}_{1}\right),\left(\mathrm{x}_{2},\left[\inf \left\{\mathrm{u}_{2}, \mathrm{u}_{3}, \mathrm{u}_{2} \ldots\right\}, \sup \left\{\mathrm{v}_{2}, \mathrm{v}_{3}, \mathrm{v}_{2} \ldots\right\}, \sup \left\{\mathrm{w}_{2}, \mathrm{w}_{3}, \mathrm{w}_{2} \ldots\right\}\right]\right.$,

$\left(\mathrm{x}_{3},\left[\inf \left\{\mathrm{u}_{3}, \mathrm{u}_{2}, \mathrm{u}_{3} \ldots\right\}, \sup \left\{\mathrm{v}_{3}, \mathrm{v}_{2}, \mathrm{v}_{3} \ldots\right\}, \sup \left\{\mathrm{w}_{3}, \mathrm{w}_{2}, \mathrm{w}_{3} \ldots\right\}\right]\right.$

$=\left(\mathrm{x}_{1}, \mathrm{u}_{1}, \mathrm{v}_{1}, \mathrm{w}_{1}\right),\left(\mathrm{x}_{2},\left[\min \left\{\mathrm{u}_{2}, \mathrm{u}_{3}\right\}, \max \left\{\mathrm{v}_{2}, \mathrm{v}_{3}\right\}, \max \left\{\mathrm{w}_{2}, \mathrm{w}_{3}\right\}\right]\right.$, 
$\left(\mathrm{x}_{3},\left[\min \left\{\mathrm{u}_{3}, \mathrm{u}_{2}\right\}, \max \left\{\mathrm{v}_{3}, \mathrm{v}_{2}\right\}, \max \left\{\mathrm{w}_{3}, \mathrm{w}_{2}\right\}\right]\right.$

$=\mu$

In general, if $X=\left\{x_{1}, x_{2}, \ldots\right\}$ and $\mu$ be a neutrosophic orbit open set under the mappingf, then there exists a neutrosophic set

$\lambda=\left\{\left(\mathrm{x}_{1}, \mathrm{u}_{1}, \mathrm{v}_{1}, \mathrm{w}_{1}\right),\left(\mathrm{x}_{2}, \mathrm{u}_{2}, \mathrm{v}_{2}, \mathrm{w}_{2}\right),\left(\mathrm{x}_{3}, \mathrm{u}_{3}, \mathrm{v}_{3}, \mathrm{w}_{3}\right) \ldots\right\}=\left\{\left(\mathrm{x}_{\mathrm{i}}, \mathrm{u}_{\mathrm{i}}, \mathrm{v}_{\mathrm{i}}, \mathrm{w}_{\mathrm{i}}\right) ; \mathrm{x}_{\mathrm{i}} \in \mathrm{X}, \mathrm{u}_{\mathrm{i}}, \mathrm{v}_{\mathrm{i}}, \mathrm{w}_{\mathrm{i}} \in \mathrm{I}, \mathrm{i} \in \wedge\right\}$

Such that $\mathrm{NO}_{\mathrm{f}}(\lambda)=\mu$. This implies

$N_{f}(\lambda)=\left\{\begin{array}{c}\left(x_{i},\left(u_{i}, v_{i}, w_{i}\right)\right) ; f\left(x_{i}\right)=x_{j}, i=j, i \in \Lambda \\ \left(x_{i},\left[\inf \left\{u_{i}\right\}, \sup \left\{v_{i}\right\}, \sup \left\{w_{i}\right\}\right],\right) ; f\left(x_{i}\right)=x_{j}, i \neq j, i \in \Lambda\end{array}\right.$
$=\left\{\begin{array}{c}\left(x_{i},\left(u_{i}, v_{i}, w_{i}\right)\right) ; f\left(x_{i}\right)=x_{j}, i=j, i \in \Lambda \\ \left(x_{i},(r, s, t)\right) ; r=\inf \left\{u_{i}\right\}, s=\sup \left\{v_{i}\right\}, t=\sup \left\{w_{i}\right\} ; f\left(x_{i}\right)=x_{j}, i \neq j, i \in \Lambda\end{array}\right.$
$=\mu$

Now for each $\mathrm{x}_{\mathrm{j}} \in \mathrm{X}$, we have

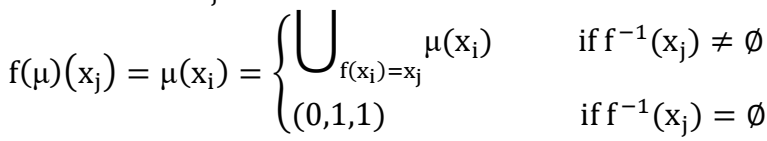

From the hypothesis and the definition of $\mathrm{f}$,we get for all $\mathrm{x}_{\mathrm{j}} \in \mathrm{X}$.

$f(\mu)\left(x_{j}\right)= \begin{cases}\left(u_{i}, v_{i}, w_{i}\right) & \text { if } i=j \\ (r, s, t), & \text { if } i \neq j\end{cases}$

Hence $f(\mu)=\mu$.

Case 3:

If $\mathrm{f}$ is the identity mapping. In this case, every open neutrosophic set in $\mathrm{X}$ is neutrosophic orbit open set under the mapping fandf $(\mu)=\mu$ for every neutrosophic set $\mu \in \mathrm{I}^{\mathrm{X}}$. Thus the proof is obtained.

Theorem 3.2:

Let $(X, \tau)$ be a NTOPand $f: X \rightarrow X$ be any constant mapping. Then $f(\mu)=\mu$ for any neutrosophic orbit open set $\mu$ under the mapping $f$.

Proof:

Let $(X, \tau)$ be a neutrosophic topological space.

Let $\mu$ be a neutrosophic orbit open set under the mapping $f$.

Then, from Definition, there exists a neutrosophic set $\lambda=\left\{\left(\mathrm{x}_{\mathrm{i}}, \mathrm{u}_{\mathrm{i}}, \mathrm{v}_{\mathrm{i}}, \mathrm{w}_{\mathrm{i}}\right) ; \mathrm{x}_{\mathrm{i}} \in \mathrm{X}, \mathrm{u}_{\mathrm{i}}, \mathrm{v}_{\mathrm{i}}, \mathrm{w}_{\mathrm{i}} \in \mathrm{I}, \mathrm{i} \in \Lambda\right\}$ such that $N O_{f}(\lambda)=\mu$.

Since $f$ is constant mapping, this implies there exists a fixed element $x_{k} \in X$ such that $f\left(x_{i}\right)=x_{k}$ for all $x_{i} \in X$ and $i \in \Lambda$.

Now form, the definition of $f(\lambda)$ for all $x_{j} \in X$, we have

$f(\lambda)\left(x_{j}\right)=\left\{\begin{array}{cl}\bigcup_{\substack{\mathrm{f}\left(\mathrm{x}_{\mathrm{i}}\right)=\mathrm{x}_{\mathrm{j}} \\(0,1,1)}} \lambda\left(\mathrm{x}_{\mathrm{i}}\right) & \text { if } f^{-1}\left(x_{j}\right) \neq \emptyset \\ (0,1) \text { orwise }\end{array}\right.$

Thus

$f(\lambda)\left(x_{j}\right)= \begin{cases}{\left[\sup _{i \in \wedge}\left\{\lambda\left(x_{i}\right)\right\}, \inf _{i \in \Lambda}\left\{\lambda\left(x_{i}\right)\right\}, \inf _{i \in \Lambda}\left\{\lambda\left(x_{i}\right)\right\}\right]} & \text { if } x_{j}=x_{k}, \\ (0,1,1) & \text { if } x_{j} \neq x_{k}\end{cases}$

Therefore, $f(\lambda)=\left\{\left(x_{k}, \sup _{i \in \Lambda}\left\{\lambda\left(x_{i}\right)\right\}, \inf _{i \in \Lambda}\left\{\lambda\left(x_{i}\right)\right\}, \inf _{i \in \Lambda}\left\{\lambda\left(x_{i}\right)\right\}\right)\right\}$.

This means $f(\lambda)$ is a neutrosophic point in $\mathrm{X}$ with support

$\mathrm{x}_{\mathrm{k}}$ and degree $\sup _{\mathrm{i} \in \Lambda}\left\{\lambda\left(\mathrm{x}_{\mathrm{i}}\right)\right\}$, degree $\inf _{\mathrm{i} \in \Lambda}\left\{\lambda\left(\mathrm{x}_{\mathrm{i}}\right)\right\}$, degree $\inf _{\mathrm{i} \in \Lambda}\left\{\lambda\left(\mathrm{x}_{\mathrm{i}}\right)\right\}$.

By the same way we have

$f^{2}(\lambda)=\left\{\left(x_{k}, \sup _{i \in \Lambda}\left\{\lambda\left(x_{i}\right)\right\}, \inf _{i \in \Lambda}\left\{\lambda\left(x_{i}\right)\right\}, \inf _{i \in \Lambda}\left\{\lambda\left(x_{i}\right)\right\}\right)\right\}$,

$f^{3}(\lambda)=\left\{\left(x_{k}, \sup _{i \in \Lambda}\left\{\lambda\left(x_{i}\right)\right\}, \inf _{i \in \Lambda}\left\{\lambda\left(x_{i}\right)\right\}\right), \inf _{i \in \Lambda}\left\{\lambda\left(x_{i}\right)\right\}\right\}, \ldots$

For more clearing we have the following:

$\lambda=\left\{\left(x_{1}, u_{1}, v_{1}, w_{1}\right),\left(x_{2}, u_{2}, v_{2}, w_{2}\right), \ldots,\left(x_{k}, u_{k}, v_{k}, w_{k}\right), \ldots\right\}$

$f(\lambda)=\left\{\left(x_{1}, 0,1,1\right),\left(x_{2}, 0,1,1\right), \ldots,\left(x_{k}, \sup _{i \in \Lambda}\left\{\lambda\left(x_{i}\right)\right\}, \inf _{i \in \Lambda}\left\{\lambda\left(x_{i}\right)\right\}, \inf _{i \in \Lambda}\left\{\lambda\left(x_{i}\right)\right\}\right), \ldots\right\}$

$f^{2}(\lambda)=\left\{\left(x_{1}, 0,1,1\right),\left(x_{2}, 0,1,1\right), \ldots,\left(x_{k}, \sup _{i \in \Lambda}\left\{\lambda\left(x_{i}\right)\right\}, \inf _{i \in \Lambda}\left\{\lambda\left(x_{i}\right)\right\}, \inf _{i \in \Lambda}\left\{\lambda\left(x_{i}\right)\right\}\right), \ldots\right\}$

$\left.f^{3}(\lambda)=\left\{\left(x_{1}, 0,1,1\right),\left(x_{2}, 0,1,1\right), \ldots,\left(x_{k}, \sup _{i \in \wedge}\left\{\lambda\left(x_{i}\right)\right\}, \inf _{i \in \Lambda}\left\{\lambda\left(x_{i}\right)\right\}, \inf _{i \in \Lambda}\left\{\lambda\left(x_{i}\right)\right\}\right\}\right), \ldots\right\}$ 


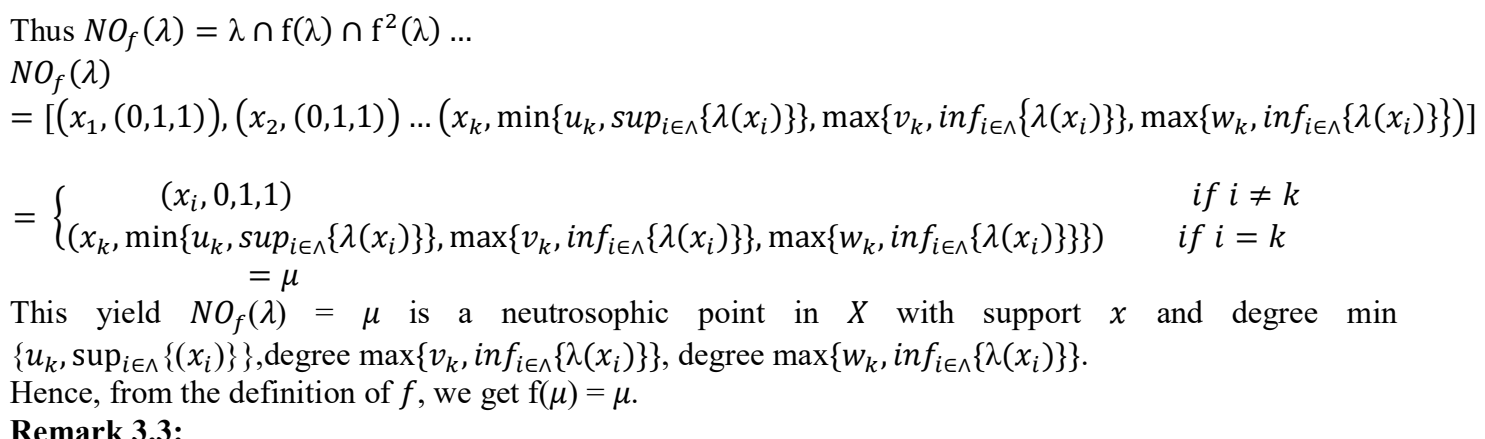

The condition to be $f: X \rightarrow X$ is bijective or constant is necessary condition to obtain fixed neutrosophic orbit open sets for any neutrosophic orbit open set $\mu$ under the mapping $f$.

For more explain, we give an example for a neutrosophic topological space $(X$,$) and f: X \rightarrow X$ not bijective, we show that $(\mu) \neq \mu$ for some neutrosophic orbit open set $\mu$ under the mapping $f$.

\section{Example 3.4:}

Let $\mathrm{X}=\left\{x_{1}, x_{2}, x_{3}, x_{4}, x_{5}\right\}$.Define $\tau=\{\overline{0}, \overline{1}, \mu\}$ where $\mu \in I^{X}$ defined as

$\mu=\left\{\left(x_{1},(0,1,1)\right),\left(x_{2},(0.1,0.4,0.9)\right),\left(x_{3},(0,0.8,1)\right),\left(x_{4},(0.6,0.2,0.4)\right),\left(x_{5},(0.6,0.2,0.4)\right)\right\}$

Define $f: X \rightarrow X$ as $f\left(x_{1}\right)=x_{2}, f\left(x_{2}\right)=x_{1}, f\left(x_{3}\right)=x_{2}, f\left(x_{4}\right)=x_{5}, f\left(x_{5}\right)=x_{4}$. It is clear that $f$ is not bijective mapping(i.e., $f$ is not one to one and not onto).Let $\lambda \in I^{X}$ defined as follows:

$\lambda=\left\{\left(x_{1}, 0.1,0.3,0.9\right),\left(x_{2}, 0.2,0.4,0.8\right),\left(x_{3}, 0,0.8,1\right),\left(x_{4}, 0.6,0.2,0.4\right),\left(x_{5}, 0.7,0.1,0.3\right)\right\}$.

Then the neutrosophic orbit of $\lambda$ are $\mathrm{NO}_{\mathrm{f}}(\lambda)=\lambda \cap \mathrm{f}(\lambda) \cap \mathrm{f}^{2}(\lambda) \ldots=\mu$. which is

$f(\lambda)=\left\{\left(x_{1}, 0,1,1\right),\left(x_{2}, 0.1,0.3,0.9\right),\left(x_{3}, 0.2,0.4,0.8\right),\left(x_{4}, 0.7,0.1,0.3\right),\left(x_{5}, 0.6,0.2,0.4\right)\right\}$

$f^{2}(\lambda)=\left\{\left(x_{1}, 0,1,1\right),\left(x_{2}, 0.2,0.4,0.8\right),\left(x_{3}, 0.1,0.3,0.9\right),\left(x_{4}, 0.6,0.2,0.4\right),\left(x_{5}, 0.7,0.1,0.3\right)\right\}$

$f^{3}(\lambda)=\left\{\left(x_{1}, 0,1,1\right),\left(x_{2}, 0.1,0.3,0.9\right),\left(x_{3}, 0.2,0.4,0.8\right),\left(x_{4}, 0.7,0.1,0.3\right),\left(x_{5}, 0.6,0.2,0.4\right)\right\}$

Therefore, the neutrosophic orbit set of $\lambda$ is $\mathrm{NO}_{\mathrm{f}}(\lambda)=\lambda \cap \mathrm{f}(\lambda) \cap \mathrm{f}^{2}(\lambda) \ldots=\mu$.

$\mathrm{NO}_{\mathrm{f}}(\lambda)=\left\{\left(x_{1},[\inf \{0.1,0,0,0 \ldots\}, \sup \{0.3,1,1, \ldots\}, \sup \{0.9,1,1, \ldots\}]\right.\right.$,

$\left(x_{2},[\inf \{0.2,0.1,0.2,0.1, \ldots\}, \sup \{0.4,0.3,0.4 \ldots\}, \sup \{0.8,0.9,0.8 \ldots\}]\right.$,

$\left(x_{3},[\inf \{0,0.2,0.1,0.2, \ldots\}, \sup \{0.8,0.4,0.3,0.4, \ldots\}, \sup \{1,0.8,0.9,0.8, \ldots\}]\right.$,

$\left(x_{4},[\inf \{0.6,0.7,0.6,0.7, \ldots\}, \sup \{0.2,0.1,0.2,0.1, \ldots\}, \sup \{0.4,0.3,0.4,0.3, \ldots\}]\right.$,

$\left(x_{5},[\inf \{0.7,0.6,0.7,0.6, \ldots\}, \sup \{0.1,0.2,0.1,0.2, \ldots\}, \sup \{0.3,0.4,0.3,0.4 \ldots\}]\right.$,

$=$

$\left\{\left(x_{1},(0,1,1)\right),\left(x_{2},(0.1,0.4,0.9)\right),\left(x_{3},(0,0.8,1)\right),\left(x_{4},(0.6,0.2,0.4)\right),\left(x_{5},(0.6,0.2,0.4)\right)\right\}=\mu$

Thus, the open neutrosophic set $\mu$ is neutrosophic orbit open set under the mapping $\mathrm{f}$. $\mathrm{But} f(\mu) \neq \mu$. From Theorem 3.1 and 3.2 we obtain the following result

Result 3.5:

Let $(X, \tau)$ be a NTOPand $f: X \rightarrow X$ be any mapping such that either $f$ is bijective mapping or $f$ is constant mapping and $\mu$ is a neutrosophic orbit open set under the mapping $f$, then $f(\mu)=\mu$.

In our work, we consider the mapping $f: X \rightarrow X$ that satisfies the conditions this Result.

Proposition 3.6:

Let $(X, \tau)$ be a NTOPand $f: X \rightarrow X$ be any mapping. If $\mu$ is a neutrosophic orbit open set under the mapping $f$, then $N O_{f}(\mu)=\mu$.

Proof:

The proof follows directly from the definition of $N O_{f}(\mu)$ and Result 3.5. i.e.,

$N O_{f}(\mu)=\mu \cap f(\mu) \cap f^{2}(\mu) \cap \ldots$

From Result 3.5, we have $\mathrm{f}(\mu)=\mu$, this implies $f^{2}(\mu)=\mathrm{f}(\mathrm{f}(\mu))=\mu, f^{3}(\mu)=f\left(f^{2}(\mu)\right)=\mu, \ldots$ Hence,

$N O_{f}(\mu)=\mu$.

Theorem 3.7:

Let $(X, \tau)$ be a neutrosophic topological space and $f: X \rightarrow X$ be a mapping.

If $\mu_{1}$ and $\mu_{2}$ are neutrosophic orbit open sets under the mapping $f$, then $N O_{f}\left(\mu_{1} \cap \mu_{2}\right)=N O_{f}\left(\mu_{1}\right) \cap$ $N O_{f}\left(\mu_{2}\right)$

Proof:

First we prove the theorem if $f$ is bijective mapping. From Theorem 3.1, we have 3 cases.

We prove the theorem in case 1. 
The proof of theorem in case 2 is similar to case 1, and the prove of theorem in case 3 is easy.

Case 1:

Suppose that $f$ is bijective mapping and $f\left(x_{i}\right)=x_{j} ; x_{i}, x_{j} \in X$ and $i \neq j$ for all $i, j \in \Lambda$.

Let $\mu_{1}$ and $\mu_{2}$ are neutrosophic orbit open sets under the mapping $f$.

Then, there exist $\lambda_{1}, \lambda_{2} \in I^{X}$ defined as $\lambda_{1}=\left\{\left(x_{i}, u_{i}, v_{i}, w_{i}\right) ; x_{i} \in X, u_{i}, v_{i}, w_{i} \in I, i \in \Lambda\right\}$ and $\lambda_{2}=$ $\left\{\left(x_{i}, r_{i}, s_{i}, t_{i}\right) ; x_{i} \in X, r_{i}, s_{i}, t_{i} \in I, i \in \Lambda\right\}$ such that $N O_{f}\left(\lambda_{1}\right)=\mu_{1}$ and $N O_{f}\left(\lambda_{2}\right)=\mu_{2}$.

From Theorem 3.1 case 1 , we have $N O_{f}\left(\lambda_{1}\right)=\left\{\left(x_{i},(u, v, w)\right) ; u=\inf \left\{u_{i}, i \in \Lambda\right\}=\mu_{1}, v=\sup \left\{v_{i}, i \in\right.\right.$ $\left.\wedge\}\}=\mu_{1}, w=\sup \left\{w_{i}, i \in \wedge\right\}\right\}=\mu_{1}$

And $N O_{f}\left(\lambda_{2}\right)=\left\{\left(x_{i},(r, s, t) ; r=\inf \left\{r_{i}, i \in \wedge\right\}=\mu_{2}, s=\sup \left\{s_{i}, i \in \wedge\right\}\right\}=\mu_{2}, t=\sup \left\{t_{i}, i \in \wedge\right\}=\mu_{2}\right.$

Thus, $\mu_{1} \cap \mu_{2}=\left\{\left(x_{i},[\min \{u, r\}, \max \{v, s\}, \max \{w, t\}]\right) ; x_{i} \in X, i \in \Lambda\right\}$.

Let $\mathrm{a}=\min \{u, r\}, b=\max \{v, s\}, \mathrm{c}=\max \{w, t\}$.

Now for all $x_{j} \in X, j \in \wedge$.

$f\left(\mu_{1} \cap \mu_{2}\right)\left(x_{j}\right)= \begin{cases}\bigcup_{f\left(x_{i}\right)=x_{j}}\left(\mu_{1} \cap \mu_{2}\right)\left(x_{i}\right) & \text { if } f^{-1}\left(x_{j}\right) \neq \varnothing \\ (0,1,1) & \text { if } f^{-1}\left(x_{j}\right)=\varnothing\end{cases}$

$=(a, b, c)$

Hence $f\left(\mu_{1} \cap \mu_{2}\right)=\mu_{1} \cap \mu_{2}$. This implies $f^{2}\left(\mu_{1} \cap \mu_{2}\right)=\mu_{1} \cap \mu_{2}, f^{3}\left(\mu_{1} \cap \mu_{2}\right)=\mu_{1} \cap \mu_{2} \ldots$

Therefore, from the definition of $N O_{f}\left(\mu_{1} \cap \mu_{2}\right)$ and Theorem 3.1 we get

$N O_{f}\left(\mu_{1} \cap \mu_{2}\right)=\mu_{1} \cap \mu_{2}=N O_{f}\left(\mu_{1}\right) \cap N O_{f}\left(\mu_{2}\right)$.

Case 2:

Suppose that $f$ is bijective mapping and $\mathrm{f}\left(x_{i}\right)=x_{j} ; x_{i}, x_{j} \in X$ and $i=j$ for all $i, j \in \Lambda$.

Let $\mu_{1}$ and $\mu_{2}$ are neutrosophic orbit open sets under the mapping $f$.

Then, there exist $\lambda_{1}, \lambda_{2} \in I^{X}$ defined as $\lambda_{1}=\left\{\left(x_{i}, u_{i}, v_{i}, w_{i}\right) ; x_{i} \in X, u_{i}, v_{i}, w_{i} \in I, i \in \Lambda\right\}$ and $\lambda_{2}=\left\{\left(r_{i}, s_{i}, t_{i}\right)\right.$; $x_{i} \in X, r_{i}, s_{i}, t_{i}, \in I, i \in \Lambda$ such that $N O_{f}\left(\lambda_{1}\right)=\mu_{1}$ and $N O_{f}\left(\lambda_{2}\right)=\mu_{2}$.

From Theorem 3.1 case 2, we have

$N O_{f}\left(\lambda_{1}\right)=\left\{\left(x_{i},\left(u_{i}, v_{i}, w_{i}\right)\right) ; f\left(x_{i}\right)=x_{j}, i=j,\left(x_{i},\left[u=\inf \left\{u_{i}, i \in \wedge\right\}, v=\sup \left\{v_{i}, i \in \wedge\right\}, w=\right.\right.\right.$ $\left.\left.\left.\sup \left\{w_{i}, i \in \wedge\right\}\right]\right) ; f\left(x_{i}\right)=x_{j}, i \neq j\right\}=\mu_{1}$ and

$N O_{f}\left(\lambda_{2}\right)=\left\{\left(x_{i},\left(r_{i}, s_{i}, t_{i}\right)\right) ; f\left(x_{i}\right)=x_{j}, i=j,\left(x_{i},\left[r=\inf \left\{r_{i}, i \in \wedge\right\}, s=\sup \left\{s_{i}, i \in \wedge\right\}, t\right.\right.\right.$ $\left.\left.=\sup \left\{t_{i}, i \in \wedge\right\}\right] ; f\left(x_{i}\right)=x_{j}, i \neq j\right\}=\mu_{2}$.

Thus $\mu_{1} \cap \mu_{2}=\left\{\begin{array}{cl}\left(x_{i},\left[\min \left\{u_{i}, r_{i}\right\}, \max \left\{v_{i}, s_{i}\right\}, \max \left\{w_{i}, t_{i}\right\}\right]\right) ; & f\left(x_{i}\right)=x_{j}, i=j, \\ \left(x_{i},[\min \{u, r\}, \max \{v, s\}, \max \{w, t\}]\right) ; & f\left(x_{i}\right)=x_{j}, i \neq j\end{array}\right\}$.

Let $\mathrm{k}=\min \left\{m_{i}, s_{i}\right\}, t=\max \left\{n_{i}, r_{i}\right\}$. Now for all $x_{j} \in X, j \in \Lambda$

$f\left(\mu_{1} \cap \mu_{2}\right)\left(x_{j}\right)= \begin{cases}\bigcup_{f\left(x_{i}\right)=x_{j}}\left(\mu_{1} \cap \mu_{2}\right)\left(x_{i}\right) & \text { iff } f^{-1}\left(x_{j}\right) \neq \emptyset \\ (0,1,1) & \text { if } f^{-1}\left(x_{j}\right)=\varnothing\end{cases}$

$=\left\{\begin{aligned}\left\{\left[\min \left\{u_{i}, r_{i}\right\}, \max \left\{v_{i}, s_{i}\right\}, \max \left\{w_{i}, t_{i}\right\}\right]\right\}, & \text { if } f\left(x_{i}\right)=x_{j}, i=j \\ \{[\min \{u, r\}, \max \{v, s\}, \max \{w, t\}]\}, & \text { if } f\left(x_{i}\right)=x_{j}, i \neq j\end{aligned}\right.$

Hence $f\left(\mu_{1} \cap \mu_{2}\right)=\mu_{1} \cap \mu_{2}$.

This implies $f^{2}\left(\mu_{1} \cap \mu_{2}\right)=\mu_{1} \cap \mu_{2}, f^{3}\left(\mu_{1} \cap \mu_{2}\right)=\mu_{1} \cap \mu_{2} \ldots$

Therefore, from the definition of $N O_{f}\left(\mu_{1} \cap \mu_{2}\right)$ and Theorem 3.1 we get

$N O_{f}\left(\mu_{1} \cap \mu_{2}\right)=\mu_{1} \cap \mu_{2}=N O_{f}\left(\mu_{1}\right) \cap N O_{f}\left(\mu_{2}\right)$.

Case 3:

Now, if $f$ is constant mapping, let $\mu_{1}$ and $\mu_{2}$ are neutrosophic orbit open sets under the mapping $f$.

Then, there exist $\lambda_{1}, \lambda_{2} \in I^{X}$ defined as $\lambda_{1}=\left\{\left(x_{i}, u_{i}, v_{i}, w_{i}\right) ; \quad x_{i} \in X, u_{i}, v_{i}, w_{i} \in I, i \in \Lambda\right\}$ and $\lambda_{2}=$ $\left\{\left(x_{i}, r_{i}, s_{i}, t_{i}\right) ; x_{i} \in X, r_{i}, s_{i}, t_{i} \in I, i \in \Lambda\right\}$ such that $N O_{f}\left(\lambda_{1}\right)=\mu_{1}$ and $N O_{f}\left(\lambda_{2}\right)=\mu_{2}$.

From Theorem 3.2, we have

$N O_{f}\left(\lambda_{1}\right)$

$=\left\{\begin{array}{cc}\left(x_{i}, 0,1,1\right) & \text { if } i \neq k \\ \left.\left(x_{k},\left[\min \left\{\left(u_{k}\right), \sup _{i \in \Lambda}\left\{\lambda_{1}\left(x_{i}\right)\right\}\right\}, \max \left\{\left(v_{k}\right), \text { inf }_{i \in \Lambda}\left\{\lambda_{1}\left(x_{i}\right)\right\}\right\}, \max \left\{\left(w_{k}\right), \text { inf }_{i \in \Lambda}\left\{\lambda_{1}\left(x_{i}\right)\right\}\right\}\right]\right]\right) & \text { if } i=k\end{array}\right.$

$=\mu_{1}$

$N O_{f}\left(\lambda_{2}\right)$

$=\left\{\begin{array}{cc}\left(x_{i}, 0,0,0\right) & \text { if } i \neq k \\ \left.\left(x_{k},\left[\min \left\{\left(r_{k}\right), \sup _{i \in \wedge}\left\{\lambda_{1}\left(x_{i}\right)\right\}\right\}, \max \left\{\left(s_{k}\right), \inf _{i \in \Lambda}\left\{\lambda_{1}\left(x_{i}\right)\right\}\right\}, \max \left\{\left(t_{k}\right), \inf _{i \in \wedge}\left\{\lambda_{1}\left(x_{i}\right)\right\}\right\}\right]\right]\right) & \text { if } i=k\end{array}\right.$ 
$=\mu_{2}$

Thus

$\mu_{1} \cap \mu_{2}$

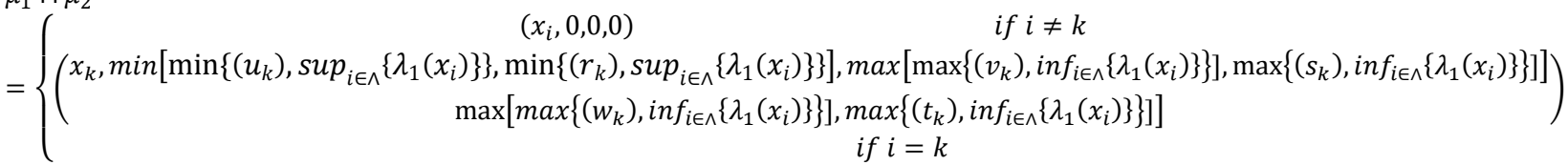

Thismeans that $\mu_{1} \cap \mu_{2}$ is a neutrosophic point in $\mathrm{X}$ with support $x_{k}$ and degree $\left[\min \left[\min \left\{\left(\mathrm{u}_{\mathrm{k}}\right), \sup _{\mathrm{i} \in \Lambda}\left\{\lambda_{1}\left(\mathrm{x}_{\mathrm{i}}\right)\right\}\right\}\right],\left[\min \left\{\left(\mathrm{r}_{\mathrm{k}}\right), \sup _{\mathrm{i} \in \Lambda}\left\{\lambda_{2}\left(\mathrm{x}_{\mathrm{i}}\right)\right\}\right\}\right]\right]$,

$\left[\max \left[\max \left\{\left(\mathrm{v}_{\mathrm{k}}\right), \inf _{\mathrm{i} \in \Lambda}\left\{\lambda_{1}\left(\mathrm{x}_{\mathrm{i}}\right)\right\}\right\}, \max \left\{\left(\mathrm{s}_{\mathrm{k}}\right), \inf _{\mathrm{i} \in \wedge}\left\{\lambda_{1}\left(\mathrm{x}_{\mathrm{i}}\right)\right\}\right\}\right],\left[\max \left[\max \left\{\left(\mathrm{w}_{\mathrm{k}}\right), \inf _{\mathrm{i} \in \wedge}\left\{\lambda_{1}\left(\mathrm{x}_{\mathrm{i}}\right)\right\}\right\}\right], \max \left\{\left(\mathrm{t}_{\mathrm{k}}\right), \inf _{\mathrm{i} \in \Lambda}\left\{\lambda_{2}\left(\mathrm{x}_{\mathrm{i}}\right)\right\}\right\}\right]\right]$

Hence from the definition of $f$ we $\operatorname{getf}\left(\mu_{1} \cap \mu_{2}\right)=\mu_{1} \cap \mu_{2}$. This implies $\mathrm{f}^{2}\left(\mu_{1} \cap \mu_{2}\right)=\mu_{1} \cap \mu_{2}$,

$\mathrm{f}^{3}\left(\mu_{1} \cap \mu_{2}\right)=\mu_{1} \cap \mu_{2} \ldots$

Therefore, from the definition of $\mathrm{NO}_{\mathrm{f}}\left(\mu_{1} \cap \mu_{2}\right)$ and Theorem 3.2 we get

$\mathrm{NO}_{\mathrm{f}}\left(\mu_{1} \cap \mu_{2}\right)=\mu_{1} \cap \mu_{2}=\mathrm{NO}_{\mathrm{f}}\left(\mu_{1}\right) \cap \mathrm{NO}_{\mathrm{f}}\left(\mu_{2}\right)$.

Hence the proof

Theorem 3.8:

Let $(X, \tau)$ be a NTOPand $f: X \rightarrow X$ be a mapping.Let $\left\{\mu_{\alpha}\right\}_{\alpha \in \Delta}$ be any family of neutrosophic orbit open sets under the mapping f,then $\mathrm{NO}_{\mathrm{f}}\left(\mathrm{U}_{\alpha \in \Delta} \mu_{\alpha}\right)=\mathrm{U}_{\alpha \in \Delta} \mathrm{NO}_{\mathrm{f}}\left(\mu_{\alpha}\right)$

Proof:

The outline of proofing this theorem is proceeds in a way similar to Theorem 3.7. As in Theorem 3.7, we consider 3 cases:

Case 1:

Suppose that $f$ is bijective mapping

andf $\left(x_{i}\right)=x_{j} ; x_{i}, x_{j} \in X$ and $i \neq j$ for all $i, j \in \Lambda$.

Let $\left\{\mu_{\alpha}\right\}_{\alpha \in \Delta}$ be any family of neutrosophic orbit open sets under the mapping f,then there exists $\lambda_{\alpha} \in$ $\mathrm{I}^{\mathrm{X}}, \alpha \in \Delta$ defined as $\lambda_{\alpha}=\left\{\left(\mathrm{x}_{\mathrm{i}}, \mathrm{u}_{\mathrm{i}_{\alpha}}, \mathrm{v}_{\mathrm{i}_{\alpha}}, \mathrm{w}_{\mathrm{i}_{\alpha}}\right) ; \mathrm{x}_{\mathrm{i}} \in \mathrm{X}, \mathrm{u}_{\mathrm{i}_{\alpha}}, \mathrm{v}_{\mathrm{i}_{\alpha}}, \mathrm{w}_{\mathrm{i}_{\alpha}} \in \mathrm{I}, \mathrm{i} \in \Lambda\right\}$ such that $\mathrm{NO}_{\mathrm{f}}\left(\lambda_{\alpha}\right)=$ $\mu_{\alpha}$ for all $\alpha \in \Delta$. From Theorem 3.1 case 1 , we have

$N O_{f}\left(\lambda_{\alpha}\right)=\left\{\left(x_{i},\left(u_{\alpha}, v_{\alpha}, v_{\alpha}\right)\right) ; u_{\alpha}=\inf \left\{u_{i_{\alpha}}, i \in \wedge\right\}, v_{\alpha}=\sup \left\{v_{i_{\alpha}}, i \in \wedge\right\}, w_{\alpha}=\sup \left\{w_{i_{\alpha}}, i \in \wedge\right\}\right\}=\mu_{\alpha}$

Thus $\left(\mathrm{U}_{\alpha \in \Delta} \mu_{\alpha}\right)=\left\{\left(\mathrm{x}_{\mathrm{i}},\left[\sup _{\alpha \in \Delta}\left(\mathrm{u}_{\alpha}\right)\right], \inf _{\alpha \in \Delta}\left[\left(\mathrm{v}_{\alpha}\right)\right], \inf _{\alpha \in \Delta}\left[\left(\mathrm{w}_{\alpha}\right) ; \mathrm{x}_{\mathrm{i}} \in \mathrm{X}, \mathrm{i} \in \Lambda\right\}\right.\right.$.

Let $a=\sup _{\alpha \in \Delta}\left(u_{\alpha}\right), b=\inf _{\alpha \in \Delta}\left(v_{\alpha}\right), c=\inf _{\alpha \in \Delta}\left(w_{\alpha}\right)$. Now for all $x_{j} \in X, j \in \Lambda$.

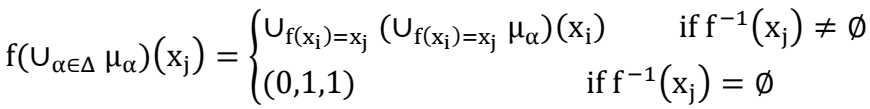

$=(\mathrm{a}, \mathrm{b}, \mathrm{c})$

Hence $\mathrm{f}\left(\mathrm{U}_{\alpha \in \Delta} \mu_{\alpha}\right)=\left(\mathrm{U}_{\alpha \in \Delta} \mu_{\alpha}\right)$. This implies $\mathrm{f}^{2}\left(\mathrm{U}_{\alpha \in \Delta} \mu_{\alpha}\right)=\left(\mathrm{U}_{\alpha \in \Delta} \mu_{\alpha}\right), \mathrm{f}^{3}\left(\mathrm{U}_{\alpha \in \Delta} \mu_{\alpha}\right)=\left(\mathrm{U}_{\alpha \in \Delta} \mu_{\alpha}\right), \ldots$

Therefore from the definition of $\mathrm{NO}_{\mathrm{f}}\left(\mathrm{U}_{\alpha \in \Delta} \mu_{\alpha}\right)$ and Theorem 3.1 we get

$N O_{f}\left(\mathrm{U}_{\alpha \in \Delta} \mu_{\alpha}\right)=\left(\mathrm{U}_{\alpha \in \Delta} \mu_{\alpha}\right)=\mathrm{U}_{\alpha \in \Delta} N O_{f}\left(\mu_{\alpha}\right)$

Case 2:

Suppose that $f$ is bijective mapping and $f\left(x_{i}\right)=x_{j} ; x_{i}, x_{j} \in X$ and $i=j$ for all $i, j \in \Lambda$.

Let $\left\{\mu_{\alpha}\right\}_{\alpha \in \Delta}$ be any family of neutrosophic orbit open sets under the mapping $f$, then there exists $\lambda_{\alpha} \in$ $\mathrm{I}^{\mathrm{X}}, \alpha \in \Delta$ defined as $\lambda_{\alpha}=\left\{\left(\mathrm{x}_{\mathrm{i}}, \mathrm{u}_{\mathrm{i}_{\alpha}}, \mathrm{v}_{\mathrm{i}_{\alpha}}, \mathrm{w}_{\mathrm{i}_{\alpha}}\right) ; \mathrm{x}_{\mathrm{i}} \in \mathrm{X}, \mathrm{u}_{\mathrm{i}_{\alpha}}, \mathrm{v}_{\mathrm{i}_{\alpha}}, \mathrm{w}_{\mathrm{i}_{\alpha}} \in \mathrm{I}, \mathrm{i} \in \Lambda\right\}$ such that $\mathrm{NO}_{\mathrm{f}}\left(\lambda_{\alpha}\right)=$ $\mu_{\alpha}$ for all $\alpha \in \Delta$. From Theorem 3.1 case 2 we have

$\mathrm{NO}_{\mathrm{f}}\left(\lambda_{\alpha}\right)=\left\{\left(\mathrm{x}_{\mathrm{i}},\left(\mathrm{u}_{\mathrm{i}_{\alpha}}, \mathrm{v}_{\mathrm{i}_{\alpha}}, \mathrm{w}_{\mathrm{i}_{\alpha}}\right)\right) ; \mathrm{f}\left(\mathrm{x}_{\mathrm{i}}\right)=\mathrm{x}_{\mathrm{j}}, \mathrm{i}=\mathrm{j},\left(\mathrm{x}_{\mathrm{i}},\left[\inf \left\{\mathrm{u}_{\mathrm{i}_{\alpha}}\right\}, \sup \left\{\mathrm{v}_{\mathrm{i}_{\alpha}}\right\}, \sup \left\{\mathrm{w}_{\mathrm{i}_{\alpha}}\right\}\right]\right.\right.$

put $\mathrm{u}_{\alpha}=\inf \left\{\mathrm{u}_{\mathrm{i}_{\alpha}}, \mathrm{i} \in \Lambda\right\}, \mathrm{v}_{\alpha}=\sup \left\{\mathrm{v}_{\mathrm{i}_{\alpha}}, \mathrm{i} \in \Lambda\right\}, \mathrm{w}_{\alpha}=\sup \left\{\mathrm{w}_{\mathrm{i}_{\alpha}}, \mathrm{i} \in \Lambda\right\}$, it follows:

$\left(\mathrm{u}_{\alpha \in \Delta} \mu_{\alpha}\right)=\left\{\left(\mathrm{x}_{\mathrm{i}}, \sup _{\alpha \in \Delta}\left(\mathrm{u}_{\mathrm{i}_{\alpha}}\right), \inf _{\alpha \in \Delta}\left(\mathrm{v}_{\mathrm{i}_{\alpha}}\right), \inf _{\alpha \in \Delta}\left(\mathrm{w}_{\mathrm{i}_{\alpha}}\right)\right) ; \mathrm{f}\left(\mathrm{x}_{\mathrm{i}}\right)=\mathrm{x}_{\mathrm{j}}, \mathrm{i}=\mathrm{j}\right.$,

$\left.\left.\left(x_{i}, \sup _{\alpha \in \Delta}\left(u_{\alpha}\right), \inf _{\alpha \in \Delta}\left(v_{\alpha}\right), \inf _{\alpha \in \Delta}\left(w_{\alpha}\right)\right) ; i \in \Lambda\right) ; f\left(x_{i}\right)=x_{j}, i \neq j\right\}$.

Let $\mathrm{k}=\sup _{\alpha \in \Delta}\left(\mathrm{m}_{\mathrm{i}_{\alpha}}, \mathrm{n}_{\mathrm{i}_{\alpha}}\right), \mathrm{t}=\inf _{\alpha \in \Delta}\left[1-\left(\mathrm{m}_{\mathrm{i}_{\alpha}}, \mathrm{n}_{\mathrm{i}_{\alpha}}\right)\right], \mathrm{p}=\sup _{\alpha \in \Delta}\left[\inf \left\{\mathrm{m}_{\alpha}\right\}, \sup \left\{\mathrm{n}_{\alpha}\right\}\right]$,

$\mathrm{q}=\inf _{\alpha \in \Delta}\left[1-\left[\inf \left\{\mathrm{m}_{\alpha}\right\}, \sup \left\{\mathrm{n}_{\alpha}\right\}\right]\right]$

Now for all $x_{j} \in X, j \in \Lambda$

$\mathrm{f}\left(\mathrm{U}_{\alpha \in \Delta} \mu_{\alpha}\right)\left(\mathrm{x}_{\mathrm{j}}\right)$

$= \begin{cases}U_{f\left(x_{i}\right)=x_{j}}\left(U_{f\left(x_{i}\right)=x_{j}} \mu_{\alpha}\right)\left(x_{i}\right) & \text { if } f^{-1}\left(x_{j}\right) \neq \varnothing \\ (0,1,1) & \text { if } f^{-1}\left(x_{j}\right)=\varnothing\end{cases}$ 
$= \begin{cases}\sup _{\alpha \in \Delta}\left(u_{i_{\alpha}}\right), \inf _{\alpha \in \Delta}\left(v_{i_{\alpha}}\right), \inf _{\alpha \in \Delta}\left(w_{i_{\alpha}}\right) & \text { if } f\left(x_{i}\right)=x_{j}, i=j, \\ \sup _{\alpha \in \Delta}\left(u_{\alpha}\right), \inf _{\alpha \in \Delta}\left(v_{\alpha}\right), \inf _{\alpha \in \Delta}\left(w_{\alpha}\right) & \text { if } f\left(x_{i}\right)=x_{j}, i \neq j\end{cases}$

Hence $\mathrm{f}\left(\mathrm{U}_{\alpha \in \Delta} \mu_{\alpha}\right)=\left(\mathrm{U}_{\alpha \in \Delta} \mu_{\alpha}\right)$. This implies $\mathrm{f}^{2}\left(\mathrm{U}_{\alpha \in \Delta} \mu_{\alpha}\right)=\left(\mathrm{U}_{\alpha \in \Delta} \mu_{\alpha}\right), \mathrm{f}^{3}\left(\mathrm{U}_{\alpha \in \Delta} \mu_{\alpha}\right)=$

$\left(\cup_{\alpha \in \Delta} \mu_{\alpha}\right), \ldots$. Therefore from the definition of $\mathrm{NO}_{\mathrm{f}}\left(\cup_{\alpha \in \Delta} \mu_{\alpha}\right)$ and Theorem 3.1 we getf

$\mathrm{NO}_{\mathrm{f}}\left(\mathrm{U}_{\alpha \in \Delta} \mu_{\alpha}\right)=\left(\mathrm{U}_{\alpha \in \Delta} \mu_{\alpha}\right)=\mathrm{U}_{\alpha \in \Delta} \mathrm{NO}_{\mathrm{f}}\left(\mu_{\alpha}\right)$

Case 3:

Now if $\mathrm{f}$ is any constant mapping,

Let $\left\{\mu_{\alpha}\right\}_{\alpha \in \Delta}$ be any family of neutrosophic orbit open sets under the mapping $\mathrm{f}$, then there exists $\lambda_{\alpha} \in$ $\mathrm{I}^{\mathrm{X}}, \alpha \in \Delta \quad$ defined as $\lambda_{\alpha}=\left\{\left(\mathrm{x}_{\mathrm{i}}, \mathrm{u}_{\mathrm{i}_{\alpha}}, \mathrm{v}_{\mathrm{i}_{\alpha}}, \mathrm{w}_{\mathrm{i}_{\alpha}}\right) ; \mathrm{x}_{\mathrm{i}} \in \mathrm{X}, \mathrm{u}_{\mathrm{i}_{\alpha}}, \mathrm{v}_{\mathrm{i}_{\alpha}}, \mathrm{w}_{\mathrm{i}_{\alpha}} \in \mathrm{I}, \mathrm{i} \in \Lambda\right\}$ such that $\mathrm{NO}_{\mathrm{f}}\left(\lambda_{\alpha}\right)=$ $\mu_{\alpha}$ for all $\alpha \in \Delta$.

From Theorem 3.2 we get,

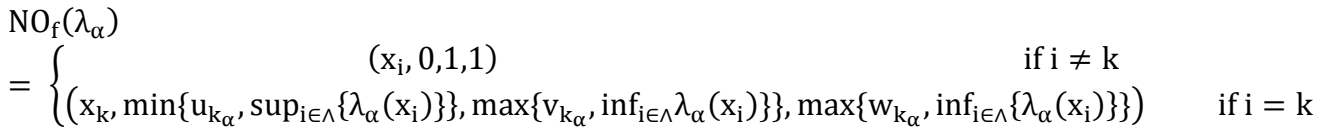

Thus,

$\left(\mathrm{U}_{\alpha \in \Delta} \mu_{\alpha}\right)$

$=\left\{\begin{array}{cc}\left(\mathrm{x}_{\mathrm{i}}, 0,1,1\right) & \text { if } \mathrm{i} \neq \mathrm{k} \\ \left(\mathrm{x}_{\mathrm{k}}, \sup _{\alpha \in \Delta}\left\{\min \left\{\mathrm{u}_{\mathrm{k}_{\alpha}}, \sup _{\mathrm{i} \in \Lambda}\left\{\lambda_{\alpha}\left(\mathrm{x}_{\mathrm{i}}\right)\right\}\right\}, \inf _{\alpha \in \Delta}\left\{\max \left\{\mathrm{v}_{\mathrm{k}_{\alpha}}, \inf _{\mathrm{i} \in \Lambda} \lambda_{\alpha}\left(\mathrm{x}_{\mathrm{i}}\right)\right\}\right\}, \inf _{\alpha \in \Delta}\left\{\max \left\{\mathrm{w}_{\mathrm{k}_{\alpha}}, \inf _{\mathrm{i} \in \Lambda}\left\{\lambda_{\alpha}\left(\mathrm{x}_{\mathrm{i}}\right)\right\}\right\}\right) \quad \text { if } \mathrm{i}=\mathrm{k}\right.\right.\end{array}\right.$

This means $\left(\mathrm{U}_{\alpha \in \Delta} \mu_{\alpha}\right)$ is a neutrosophic point in $\mathrm{X}$ with support $\mathrm{x}_{\mathrm{k}}$ and degree $\left[\sup _{\alpha \in \Delta}\left\{\min \left\{\left(\mathrm{m}_{\mathrm{k}_{\alpha}}\right), \sup _{\mathrm{i} \in \Lambda}\left\{\lambda_{\alpha}\left(\mathrm{x}_{\mathrm{i}}\right)\right\}\right\}\right\},\left\{\max \left\{\left(\mathrm{n}_{\mathrm{k}_{\alpha}}\right), \inf _{\mathrm{i} \in \Lambda}\left\{\lambda_{\alpha}\left(\mathrm{x}_{\mathrm{i}}\right)\right\}\right\}\right\}\right]$,

Hence from the definition of $\mathrm{f}$ we get $\mathrm{f}\left(\mathrm{U}_{\alpha \in \Delta} \mu_{\alpha}\right)=\left(\mathrm{U}_{\alpha \in \Delta} \mu_{\alpha}\right)$. This implies $\mathrm{f}^{2}\left(\mathrm{U}_{\alpha \in \Delta} \mu_{\alpha}\right)=\left(\mathrm{U}_{\alpha \in \Delta} \mu_{\alpha}\right)$, $\mathrm{f}^{3}\left(\mathrm{U}_{\alpha \in \Delta} \mu_{\alpha}\right)\left(\mathrm{U}_{\alpha \in \Delta} \mu_{\alpha}\right), \ldots$. Therefore from the definition of $\mathrm{NO}_{\mathrm{f}}\left(\mathrm{U}_{\alpha \in \Delta} \mu_{\alpha}\right)$ and Theorem 3.2 we get $\mathrm{NO}_{\mathrm{f}}\left(\mathrm{U}_{\alpha \in \Delta} \mu_{\alpha}\right)=\left(\mathrm{U}_{\alpha \in \Delta} \mu_{\alpha}\right)=\mathrm{U}_{\alpha \in \Delta} \mathrm{NO}_{\mathrm{f}}\left(\mu_{\alpha}\right)$

Hence the proof.

\section{Neutrosophic orbit topological spaces}

In this section we show that the family of all neutrosophic orbit open sets under the mapping $f$ constrict a neutrosophic orbit topology on $X$, denoted by $\tau_{N O}$ which is coarser than $\tau$.

\section{Theorem 4.1:}

Let $(X, \tau)$ be a NTS and $f: X \rightarrow X$ be a mapping.Let $\tau_{N O}$ denote to the family of all neutrosophic orbit open sets under the mapping f.Then, $\tau_{\mathrm{NO}}$ is a neutrosophic topology on $X$ coaser than $\tau$.

Proof:

We must show $\tau_{N O}$ satisfies the 3 axioms of the definition of neutrosophic topology. It is clear that $\overline{0}$ and $\overline{1}$ are neutrosophic orbit open sets because there exist $\lambda=\overline{0}$ and $v=\overline{1}$ such that $N O_{f}(\lambda)=\overline{0} \in \tau$ and $N O_{f}($ $v)=\overline{1} \in \tau$. Thus, $\overline{0} \in \tau_{N O}$ and $\overline{1} \in \tau_{N O}$.

Let $\mu_{1}$ and $\mu_{2}$ be neutrosophic orbit open sets under the mapping f.To show $\mu_{1} \cap \mu_{2}$ is a neutrosophic orbit open sets under the mapping $\mathrm{f}$, we must find a neutrosophic set $\lambda \in I^{X}$ such that

$$
N O_{f}(\lambda)=\mu_{1} \cap \mu_{2} \in \tau
$$

If we choose $\lambda=\mu_{1} \cap \mu_{2}$ then from Theorem 3.3 and Proposition 3.6 we have $N O_{f}(\lambda)=N O_{f}\left(\mu_{1} \cap \mu_{2}\right)=N O_{f}\left(\mu_{1}\right) \cap N O_{f}\left(\mu_{2}\right)=\mu_{1} \cap \mu_{2}$

On the other hand, since every neutrosophic orbit open set is an open neutrosophic set in $X$, then $\mu_{1} \cap$ $\mu_{2} \in \tau$

Hence, the result.

Let $\left\{\mu_{\alpha}\right\}_{\alpha \in \Delta}$ be any family of neutrosophic orbit open sets under the mapping f. Let $\lambda=\left(\cup_{\alpha \in \Delta} \mu_{\alpha}\right)$.

Then from Theorem 3.9, $N O_{f}(\lambda)=N O_{f}\left(\mathrm{~V}_{\alpha \in \Delta} \mu_{\alpha}\right)=\cup_{\alpha \in \Delta} N O_{f}\left(\mu_{\alpha}\right)=\left(\cup_{\alpha \in \Delta} \mu_{\alpha}\right)$. And $\left(\cup_{\alpha \in \Delta} \mu_{\alpha}\right) \in \tau$.

Thus, $\tau_{N O}$ is a neutrosophic topology on $X$.

Furthermore, $\tau_{N O} \subset \tau$ since every neutrosophic orbit open set is an open neutrosophic set in $X$.

Definition 4.2:

Let $(X, \tau)$ be a NTOPand $f: X \rightarrow X$ be a mapping. The pair $\left(X, \tau_{N O}\right)$ is called neutrosophic orbit topological space (NOT) associated with $(X, \tau)$ if it satisfies the following axioms

(i) $\overline{0} \in \tau_{N O}$ and $\overline{1} \in \tau_{N O}$

(ii) $\quad G_{1} \cap G_{2} \in \tau_{N O}$, forany $G_{1}, G_{2} \in \tau_{N O}$ 
(iii)

$\cup G_{i} \in \tau_{N O}$, foranyarbitraryfamily $\left\{G_{i}, G_{i} \in \tau_{N O}, i \in I\right\}$

Example 4.3:

1. For any nonempty countable set $X, \tau_{N O}^{O}=\{\overline{0}, \overline{1}\}$ is a neutrosophic orbit topology on $X$, and is called the indiscrete neutrosophic orbit topology.

2. For any nonempty countable set $X$, if $f: X \rightarrow X$ is the identity mapping, then $\tau_{N O}=\tau$

Next the notion of neutrosophic orbit closure (resp. interior) of a neutrosophic set is introduced.

Definition 4.4:

Let $\left(X, \tau_{N O}\right)$ be a neutrosophic orbit topological space and $\lambda \in I^{X}$. The neutrosophic orbit closure of $\lambda$, denoted by $c l_{N O}(\lambda)$, is the intersection of all neutrosophic orbit closed supersets under the mapping $f$ of $\lambda$. i.e.,

$c l_{N O}(\lambda)=\cap\left\{\rho \in I^{X} / \rho \supseteq \lambda, \overline{1}-\rho \in \tau_{N O}\right\}$

And, the neutrosophic orbit interior of $\lambda$, denoted by $\operatorname{int}_{N O}(\lambda)$, is the union of all neutrosophic orbit open subsets under the mapping $f$ of $\lambda$ i.e.,

int $_{N O}(\lambda)=\cup\left\{\rho \in I^{X} / \rho \subseteq \lambda, p \in \tau_{N O}\right\}$

Clearly, $\operatorname{cl}_{N O}(\lambda)$ (resp., int $\left.{ }_{N O}(\lambda)\right)$ is the smallest (resp., largest)neutrosophic orbit closed (resp., open) set under the mapping $f$ which contains (resp., contained in) $\lambda$.

Proposition 4.5:

Let $\left(X, \tau_{N O}\right)$ be a neutrosophic orbit topological space and $\lambda \in I^{X}$. Then

$\operatorname{int}_{N O}(\lambda) \subseteq \operatorname{int}(\lambda) \subseteq \lambda \subseteq \operatorname{cl}(\lambda) \subseteq \operatorname{cl}_{N O}(\lambda)$

Proof:

The proof follows directly from the fact that every neutrosophic orbit closed (resp., open) set under the mapping $f$ is closed (resp., open) neutrosophic set.

Proposition 4.6:

Let $\left(X, \tau_{N O}\right)$ be a neutrosophic orbit topological space and $\lambda, \mu \in I^{X}$. Then

1. $c l_{N O}(\overline{0})=\overline{0}$ and $c l_{N O}(\overline{1})=\overline{1}$.

2. $\lambda \subseteq c l_{N O}(\lambda)$

3. $c l_{N O}(\lambda \cup \mu)=c l_{N O}(\lambda) \cup c l_{N O}(\mu)$

4. if $\lambda \subseteq \mu$, thencl $\operatorname{No}_{N O}(\lambda) \subseteq c l_{N O}(\mu)$

5. $c l_{N O}\left(c l_{N O}(\lambda)\right)=c l_{N O}(\lambda)$

6. $\lambda$ isaneutrosophicorbitclosedsetunderthemappingfiff $\lambda=\operatorname{cl}_{\mathrm{NO}}(\lambda)$.

Proof: Straightforward

Proposition 4.7:

Let $\left(X, \tau_{N O}\right)$ be a neutrosophic orbit topological space and $\lambda, \mu \in I^{X}$.Then

1. $\operatorname{int}_{N O}(\overline{0})=\overline{0}$ and $\operatorname{int}_{N O}(\overline{1})=\overline{1}$.

2. $\operatorname{int}_{N O}(\lambda) \subseteq \lambda$.

3. $\operatorname{int}_{N O}(\lambda \cup \mu)=\operatorname{int}_{N O}(\lambda) \cup \operatorname{int}_{N O}(\mu)$

4. if $\lambda \subseteq \mu$, thenint $_{N O}(\lambda) \subseteq \operatorname{int}_{N O}(\mu)$

5. $\operatorname{int}_{N O}\left(\operatorname{int}_{N O}(\lambda)\right)=\operatorname{int}_{N O}(\lambda)$

6. $\lambda$ isaneutrosophicorbitopensetunderthemappingfiff $\lambda=\operatorname{int}_{\mathrm{NO}}(\lambda)$.

Proof: Straightforward

Theorem 4.8:

Let $\left(X, \tau_{N O}\right)$ be a neutrosophic orbit topological space and $\lambda \in I^{X}$. Then,

1. $\overline{1}-i n t_{N O}(\lambda)=c l_{N O}(\overline{1}-\lambda)$.

2. $\overline{1}-c l_{N O}(\lambda)=\operatorname{int}_{N O}(\overline{1}-\lambda)$.

Proof:

We prove that part 1 and by the similar way one can prove part 2.From Proposition 4.7 part 2 int $_{N O}(\lambda) \subseteq$ $\lambda$ so by taking the complement we have $\overline{1}-\lambda \subseteq \overline{1}-\operatorname{int}_{N O}(\lambda)$. Since $\overline{1}-\operatorname{int}_{N O}(\lambda)$ is a neutrosophic

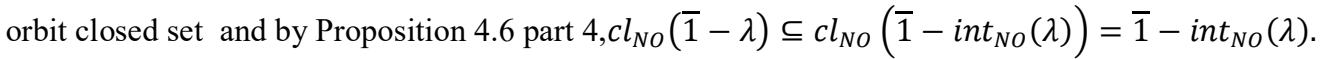

Hence $c l_{N O}(\overline{1}-\lambda) \subseteq \overline{1}-\operatorname{int}_{N O}(\lambda)$. 
Conversely, by Proposition 4.6 part $2, \overline{1}-\lambda \subseteq c l_{N O}(\overline{1}-\lambda)$. By taking the complement $\overline{1}-c l_{N O}(\overline{1}-$ $\lambda) \subseteq \lambda$. Since $c l_{N O}(\overline{1}-\lambda)$ is a neutrosophic orbit closed set.Then $\overline{1}-c l_{N O}(\overline{1}-\lambda)$ is a neutrosophic orbit open set and by Proposition 4.7 part 6 , we have $\overline{1}-c l_{N O}(\overline{1}-\lambda) \subseteq i n t_{N O}(\lambda)$, again by taking the complement we obtain,

$\overline{1}-i n t_{N O}(\lambda) \subseteq c l_{N O}(\overline{1}-\lambda)$.

Theorem 4.9:

Let $f:\left(X, \tau_{N O}\right) \rightarrow\left(Y, \tau_{N O}^{\prime}\right)$ and $g:\left(Y, \tau_{N O}\right) \rightarrow\left(Z, \tau_{N O}^{\prime \prime}\right)$ be any 2 mappings. Then $g \circ f$ is any neutrosophic continuous mapping if $f$ and $g$ are neutrosophic continuous.

Proof:

Let $\mathrm{V}$ be a orbit open set in $\left(Z, \tau_{N O}^{\prime \prime}\right)$. Then $\mathrm{g}^{-1}(\mathrm{~V})$ is neutrosophic orbit open in $\left(Y, \tau_{N O}\right)($ since $\mathrm{g}$ is neutrosophic continuous), and hence $\mathrm{f}^{-1}\left(\mathrm{~g}^{-1}(\mathrm{v})\right)$ is neutrosophic orbit open in $\left(X, \tau_{N O}\right)$ (since $\mathrm{f}$ is neutrosophic continuous). But $\mathrm{f}^{-1}\left(\mathrm{~g}^{-1}(\mathrm{v})\right)=(g \circ f)^{-1}(\mathrm{~V})$. Thus the composition function $g \circ f$ is neutrosophic continuous.

\section{Category of neutrosophic orbit topological spaces} NTOP

In this section, we will construct the category of NOTOP, and study its relation with the category of

\section{Definition5.1:}

Let NOTOPbe the collection of all $\operatorname{NOTOP}\left(X, \tau_{N O}\right),\left(Y, \tau_{N O}^{\prime}\right), \ldots$ associated with $(X, \tau),\left(Y, \tau^{\prime}\right), \ldots$ respectively. For each pair of objects $\left(X, \tau_{N O}\right),\left(Y, \tau_{N O}^{\prime}\right)$ ofNOTOP, define $\operatorname{Mor}\left(\left(X, \tau_{N O}\right),\left(Y, \tau_{N O}^{\prime}\right)\right)$ to bethe set of all neutrosophic continuous mapping $f$ with respect to $\tau_{N O}$ and $\tau_{N O}^{\prime}$. Composition of 2 morphisms $f$ : $\left(X, \tau_{N O}\right) \rightarrow\left(Y, \tau_{N O}^{\prime}\right), g:\left(Y, \tau_{N O}^{\prime}\right) \rightarrow\left(Z, \tau_{N O}^{\prime \prime}\right)$ is defined by $g o f:\left(X, \tau_{N O}\right) \rightarrow\left(\mathrm{Z}, \tau_{N O}^{\prime \prime}\right)$.

Theorem 5.2:

NOTOPis a category.

Proof:

First, from Theorem 4.9, the composition of neutrosophic continuous mappings between NOTOP is also neutrosophiccontinuous, hence the composition of morphisms is well defined and associative. Second, to each object $\left(X, \tau_{N O}\right)$ in NOTOP define the identity morphism $1_{\left(X, \tau_{N O}\right)}:\left(X, \tau_{N O}\right) \rightarrow\left(X, \tau_{N O}\right)$ by the idtity set mapping. Thus, we get to the required result.

Remark 5.3:

NOTOPis not a subcategory of NTOP, because if $f$ is intuitionistic fuzzy continuous from $\left(X, \tau_{N O}\right)$ to $\left(Y, \tau_{N O}^{\prime}\right)$, then $f$ need not to be neutrosophic continuous from $(X$,$) to \left(Y, \tau^{\prime}\right)$. That is mean $\operatorname{Mor}\left(\left(X, \tau_{N O}\right)\right.$, $\left.\left(Y, \tau_{N O}^{\prime}\right)\right) \nsubseteq \operatorname{Mor}\left((X, \tau),\left(Y, \tau^{\prime}\right)\right)$. We give an example to explain that.

\section{Example 5.4:}

Let $X=\left\{x_{1}, x_{2}, x_{3}\right\}$ and $Y=\left\{y_{1}, y_{2}, y_{3}\right\}$. Define $\tau=\{\overline{0}, \overline{1}, \lambda\}$ and $\tau^{\prime}=\left\{\overline{0}, \overline{1}, \mu_{1}, \mu_{2}\right\}$

where $\lambda \in \mathrm{I}^{\mathrm{X}}$ and $\mu_{1}, \mu_{2} \in \mathrm{I}^{\mathrm{Y}}$ suchthat $\lambda=\left\{\left(\mathrm{x}_{1}, 0.2,0.4,0.8\right),\left(\mathrm{x}_{2}, 0.3,0.5,0.7\right),\left(\mathrm{x}_{3}, 0.3,0.5,0.7\right)\right\}$,

$\mu_{1}=\left\{\left(\mathrm{y}_{1}, 0.2,0.4,0.8\right),\left(\mathrm{y}_{2}, 0.3,0.5,0.7\right),\left(\mathrm{y}_{3}, 0.3,0.5,0.7\right)\right\}$ and $\mu_{2}$

$$
=\left\{\left(\mathrm{y}_{1}, 0.6,0.3,0.4\right),\left(\mathrm{y}_{2}, 0.5,0.5,0.5\right),\left(\mathrm{y}_{3}, 0.7,0.5,0.3\right)\right\}
$$

$\operatorname{clearly}(X, \tau),\left(Y, \tau^{\prime}\right)$ areNTOP.

Definef: $(X, \tau) \rightarrow\left(Y, \tau^{\prime}\right), f_{1}: X \rightarrow X X_{n d f}: Y \rightarrow \operatorname{Yasf}\left(x_{1}\right)=y_{1}, f\left(x_{2}\right)=y_{3}, f\left(x_{3}\right)=y_{2}$,

$\mathrm{f}_{1}\left(\mathrm{x}_{1}\right)=\mathrm{x}_{1}, \mathrm{f}_{1}\left(\mathrm{x}_{2}\right)=\mathrm{x}_{3}, \mathrm{f}_{1}\left(\mathrm{x}_{3}\right)=\mathrm{x}_{2} \operatorname{andf}_{2}\left(\mathrm{x}_{1}\right)=\mathrm{x}_{1}, \mathrm{f}_{2}\left(\mathrm{x}_{2}\right)=\mathrm{x}_{3}, \mathrm{f}_{2}\left(\mathrm{x}_{3}\right)=\mathrm{x}_{2}$.

Then $\tau_{\mathrm{NO}}=\{\overline{0}, \overline{1}, \lambda\}$ and $\tau_{\mathrm{NO}}^{\prime}=\left\{\overline{0}, \overline{1}, \mu_{1}\right\}$. Itisclearthatfisaneutrosophiccontinuous

withrespectto $\tau_{\mathrm{NO}}$ and $\tau_{\mathrm{NO}}^{\prime}$.

Butfisnotaneutrosophiccontinuouswithrespecttotand $\tau^{\prime}$, since $\mu_{2}$ isanopen

neutrosophicsetinY, howeverf ${ }^{-1}\left(\mu_{2}\right)$ isnotaneutrosophicopensetinX.

Theorem 5.5:

NOTOP isomorphic to a subcategory of NTOP.

Proof:

Let NTOP $\omega$ be a collection $\left\{\left(X, I_{X}\right)\right\}$ of objects in NTOP, such that $I_{X}$ is the indiscrete neutrosophic topology on $X$. For any pair of objects $\left(X, I_{X}\right),\left(Y, I_{Y}\right)$ of NTOP $\omega$, take $\operatorname{Mor}\left(\left(X, I_{X}\right),\left(Y, I_{Y}\right)\right)$ (in NTOP) as the set of morphisms in NTOP $\omega$. Then it is clear NTOP $\omega$ is a subcategory of NTOP. Now define $F$ : NOTOP $\rightarrow \operatorname{NTOP} \omega$ by $\mathrm{F}\left(\left(X, \tau_{N O}\right)\right)=\left(X, I_{X}\right)$ and for each morphism $\mathrm{f}:\left(X, \tau_{N O}\right) \rightarrow\left(Y, \tau_{N O}^{\prime}\right)$ define $F(f)=f:\left(X, I_{X}\right)$ $\rightarrow\left(Y, I_{Y}\right)$. It can be verified that $F$ is indeed a bijective functor. Thus NOTOP isomorphic to NTOP. 
Remark 5.6: From the above theorem, we can say that NOTOP is embedded in NTOP as a subcategory.

\section{Conclusions}

In this paper, we study the collection of neutrosophic orbit open sets under the mapping $f: X \rightarrow X$. We give the necessary conditions on the mapping $f$ in order to obtain a fixed orbit of a neutrosophic set for any neutrosophic orbit open set under the mapping $f$. As a main result, we prove the family of all neutrosophic orbit open sets constructs a neutrosophic orbit topological space.In addition, the category of NOTOPand neutrosophic continuous mappings NOTOP is defined. And we show this category is isomorphic to a subcategory of the category of NTOP.

\section{References}

[1] K Atanassov. Intuitionistic fuzzy set. In: Proceedings of the VII ITKR'S session,sofia, Bulgaria. 1983.

[2] RL Devaney. Introduction to chaotic dynamical systems. Addison-wesley, Workingham, 1948.

[3] MD Priscilla and FN Irudayam. Intuitionistic fuzzy orbit topological spaces. Infokara Res. 2020; 9 , 251-66.

[4] R Malathi and MK Uma. Fuzzy orbit* continuous mappings. Ann. Fuzzy Math. Inform. 2017; 13, 465-74.

[5] RN Majeed and SA EL-sheikh. Fuzzy orbit topological spaces. Mater. Sci. Eng. 2019; 571, 012026.

[6] T Madhumathi, FN Irudayam and F Smarandache. A note on neutrosophicchaotic continuous functions. Neutrosophic Sets Syst. 2019; 25, 76-84.

[7] M Kousalyaparashakthi, E Roja and MK Uma. Intuitionistic chaotic continuousfunctions. Ann. Fuzzy Math. Inform. 2015; 9, 1-17.

[8] F Smarandache.Neutrosophy and neutrosophic logic. In: Proceedings of the $1^{\text {st }}$ International Conference onNeutrosophy, Neutrosophic Logic, Set, Probability and Statistics, New Mexico, USA. 2002.

[9] F Smarandache. A unifying field in logics: Neutrosophic logic. Neutrosophy, neutrosophi set, neutrosophic probability and statistics. American Research Press, Rehoboth, New Mexico, 1999.

[10] F Smarandache. Neutrosophic set- a generalization of intuitionistic fuzzy set. J. Def. Resour. Manag. 2010; 1, 107-16.

[11] AA Salama and SA Al-Blowi. Neutrosophic set and neutrosophic topological spaces. IOSR J. Math. 2012; 3, 31-5.

[12] AA Salama and SA Alblowi. Generalized neutrosophic set and generalized neutrosophic topological spaces. Comp. Sci. Eng. 2012; 2, 129-32.

[13] AA Salama, F Smarandache and V Kromov. Neutrosophic closed set andneutrosophic continuous functions. Neutrosophic Sets Syst. 2014; 4, 4-8.

[14] LA Zadeh. Fuzzy sets. Inf. Control 1965; 8, 338-53. 